\title{
Scleral buckling surgery for rhegmatogenous retinal detachment with subretinal proliferation
}

\begin{abstract}
Purpose To evaluate the outcome of scleral buckling surgery in patients with rhegmatogenous retinal detachment (RRD) with subretinal proliferation.

Methods In this retrospective study, a chart review of all patients with RRD associated with subretinal proliferation who were primarily treated with scleral buckling procedure, from April 2007 to April 2014, was undertaken. Main outcome measures were anatomical retinal reattachment and visual acuity.

Results Forty-four eyes of 43 patients including 24 males and 19 females with a mean age of $26.5 \pm 13.1$ years were evaluated. Immediately after the surgery, retina was reattached in all eyes. However, five eyes $\mathbf{( 1 1 . 3 \% )}$ needed additional surgery for retinal redetachment. Single surgery anatomical

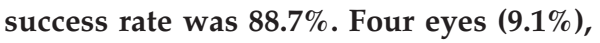
needed pars plana vitrectomy for the treatment of redetachment associated with proliferative vitreoretinopathy and scleral buckle revision surgery was successfully performed in the other eye. Best corrected visual acuity improved from $1.5 \pm 0.9 \log$ MAR before surgery to $1.1 \pm 0.7 \log$ MAR after surgery $(P<0.001)$. An improvement in BCVA of $>2$ lines was found in 23 eyes $(52.2 \%)$ and worsening of best corrected visual acuity of $>2$ lines was observed in 2 eyes (4.5\%). Conclusions Scleral buckling surgery is highly successful in eyes with RRD associated with subretinal proliferation. Eye (2015) 29, 509-514; doi:10.1038/ eye.2014.341; published online 23 January 2015
\end{abstract}

\section{Introduction}

Treatment of rhegmatogenous retinal detachment (RRD) remains controversial. Different results
K Ghasemi Falavarjani ${ }^{1}$, SA Alemzadeh², M Modarres ${ }^{1}$, MM Parvaresh ${ }^{1}$, M Hashemi ${ }^{1}$, M Naseripour ${ }^{1}$, H Nazari Khanamiri ${ }^{3}$ and S Askari ${ }^{2}$

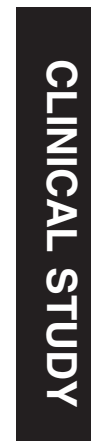

have been reported by the studies comparing scleral buckling procedure with pars plana vitrectomy. Most studies have reported comparable anatomical and visual outcomes in patients with uncomplicated RRD treated by vitrectomy or scleral buckling. ${ }^{1-5}$ In patients with complex RRD, however, pars plan vitrectomy with or without scleral buckling surgery has been associated with significantly higher success rate compared with scleral buckling alone. ${ }^{6}$

Proliferative vitreoretinopathy (PVR) is a wound healing process characterized by migration and proliferation of resident ocular cells including retinal pigment epithelial cells and invading immune cells leading to formation of adherent membranes over and under the retina. These membranes are able to contract and cause recurrent retinal detachment. ${ }^{7}$ Based on the current accepted classification of PVR, the presence of full thickness folds or subretinal strands is considered as advanced PVR (PVR C). ${ }^{8}$ Removal of contracting preretinal membranes is generally needed for a successful retinal reattachment surgery in patients with advanced PVR and pars plana vitrectomy is currently the standard of care in these patients. Subretinal proliferations also known as subretinal strands, subretinal membranes, or subretinal fibrosis are an infrequently discussed subtype of PVR. ${ }^{9,10}$ Although subretinal proliferation is classified as grade C PVR, many patients can be treated successfully by scleral buckling surgery. ${ }^{11,12}$

The aim of this study was to evaluate the outcome of scleral buckling surgery in patients with RRD associated with subretinal proliferation.

\section{Materials and methods}

In this retrospective study, the charts of all patients with RRD and subretinal proliferation
${ }^{1}$ Eye Research Center, Eye Department, Rassoul Akram Hospital, Iran University of Medical Sciences, Tehran, Iran

${ }^{2}$ Medical Student Research Committee, Iran University of Medical Sciences, Tehran, Iran

${ }^{3}$ USC Eye Institute, Los Angeles, CA, USA

Correspondence:

K Ghasemi Falavarjani, Eye Research Center, Rassoul Akram Hospital, Sattarkhan-Niayesh Street, Tehran 14456-13131, Iran Tel: +989121725850; Fax: +982166509162 E-mail: drghasemi@ yahoo.com

Received: 18 October 2014 Accepted in revised form: 19 December 2014 Published online: 23 January 2015 
who were primarily treated with scleral buckling procedure in the Rassoul Akram Hospital, from April 2007 to April 2014, were reviewed. Patients with retinal breaks posterior to equator, retinal star folds more than $\mathrm{CA} 1^{8}$ and those with napkin ring subretinal proliferation ${ }^{13}$ were excluded. Also excluded were the patients with uveitis, diabetic retinopathy, significant cataract precluding visualization of fundus details, choroidal detachment, and those with history of globe laceration repair. The study was approved by Eye Research Center Ethics Committee.

Data extracted were age, sex, refraction, duration of symptoms, location and quadrants of retinal detachment, extent of subretinal proliferation, number of retinal breaks, type of scleral buckle inserted (circumferential or segmental), anatomic outcome of surgery, need for additional surgery, and pre and postoperative best corrected visual acuity (BCVA). Visual acuity was measured using a Snellen chart and the measurements were converted to the $\log$ MAR. ${ }^{14}$ Because postoperative redetachment with PVR usually occurs within 3 months after surgery, patients had to have at least 3 months of follow up. . 2,15

Data analysis was performed using a SPSS software (version 15, SPSS Inc., Chicago, IL, USA). Student's $t$-test, paired $t$-test, $\chi^{2}$-test, and Fisher's exact test were used for analysis. $P$-value $<0.05$ was considered significant.

\section{Results}

Forty-four eyes of 43 patients including 24 males and 19 females with a mean age of $26.5 \pm 13.1$ years (range: 6-68 years) were assessed. The patients were followed for a mean of $15.8 \pm 13.9$ months (range: 3-60 months). Table 1 shows the characteristics of the patients.

Mean duration of the symptoms was $8.1 \pm 15.1$ months (range: $0.25-72$ months). Eleven eyes (25\%) had a history of blunt ocular trauma and three patients $(6.8 \%)$ were aphakic. Retinal detachment involved one quadrant in 9 eyes (20.4\%), two quadrants in 20 eyes (45.4\%), three quadrants in 7 eyes $(15.9 \%)$, and total retinal detachment was found in 8 eyes $(18.1 \%)$. Retinal detachment was found inferiorly in 5 eyes $(11.3 \%)$, temporally in 3 eyes $(6.8 \%)$, nasally in one eye $(2.3 \%)$, inferonasally in 5 eyes $(11.3 \%)$, and inferotemporally in 15 eyes (34.1\%). Also, retinal detachment was in inferior-nasal-temporal and inferior-temporal-superior quadrants in six eyes (13.6\%) and one eye $(2.3 \%)$, respectively. Macula was attached in six eyes (13.6\%). Twenty-seven eyes $(61.3 \%)$ had only one retinal break, four eyes $(9.1 \%)$ had dialysis, and the remaining eyes $(29.6 \%)$ had more than one retinal break.

Subretinal proliferation was observed in one quadrant in 26 eyes $(59.1 \%)$, two quadrants in 16 eyes $(36.4 \%)$, and three quadrants in 2 eyes (4.5\%). The extent of the subretinal proliferation was anterior to the equator in 20 eyes (45.4\%), and posterior to the equator in 6 eyes (13.6\%). Other eyes (41\%) had subretinal proliferation extended from anterior to the posterior to the equator. Seven eyes (15.9\%) had PVR grade CA1. Retina cysts were seen in five eyes (11.4\%).

Segmental buckle with encircling band (No. 240, FCI Inc., Paris, France) was inserted in 23 eyes (52.3\%), segmental buckle alone in 18 eyes $(40.9 \%)$, and encircling buckle alone in 3 eyes $(6.8 \%)$. Cryotherapy was performed

Table 1 Demographics of the patients who underwent scleral buckling surgery for retinal detachment associated with subretinal proliferation

\begin{tabular}{|c|c|c|c|c|}
\hline & Total & Single surgery success & Primary failure with $P V R$ & P-value \\
\hline Number of eyes & 44 & 39 & 4 & \\
\hline Age (year; mean \pm SD) & $26.5 \pm 13.1$ & $25.3 \pm 12.1$ & $31.2 \pm 24.9$ & $0.70^{\mathrm{a}}$ \\
\hline Sex (female/male; number) & $19 / 24$ & $18 / 22$ & $2 / 2$ & $1.00^{\mathrm{b}}$ \\
\hline Duration of symptoms (months; mean $\pm \mathrm{SD}$ ) & $8.1 \pm 15.1$ & $8.9 \pm 15.8$ & $1.7 \pm 0.9$ & $0.40^{\mathrm{a}}$ \\
\hline Baseline BCVA (LogMAR; mean \pm SD) & $1.5 \pm 0.9$ & $1.5 \pm 0.9$ & $1.9 \pm 0.7$ & $0.47^{\mathrm{a}}$ \\
\hline Baseline refraction (Diopters; mean \pm SD) & $-2.00 \pm 4.6$ & $-1.3 \pm 4.1$ & $-1.5 \pm 2.3$ & $0.94^{\mathrm{a}}$ \\
\hline Positive history of trauma (number) & 11 & 9 & 2 & $0.25^{\mathrm{b}}$ \\
\hline Total retinal detachment (number) & 8 & 6 & 2 & $0.14^{\mathrm{b}}$ \\
\hline $\begin{array}{l}\text { Extent of subretinal proliferation } \\
\text { (1 quadrant/ } 2 \text { quadrants/3 quadrants; number) }\end{array}$ & $26 / 16 / 2$ & $23 / 15 / 2$ & $3 / 1 / 0$ & $0.74^{\mathrm{c}}$ \\
\hline Number of the breaks $(1 / \geq 2$; number $)$ & $27 / 17$ & $26 / 14$ & $1 / 3$ & $0.28^{\mathrm{b}}$ \\
\hline Presence of PVR CA1 (number) & 7 & 7 & 0 & $1.00^{\mathrm{b}}$ \\
\hline Presence of retinal cyst (number) & 5 & 5 & 0 & $1.00^{\mathrm{b}}$ \\
\hline Encircling surgery (number) & 26 & 22 & 4 & $0.13^{\mathrm{b}}$ \\
\hline
\end{tabular}

Abbreviations: BCVA, best corrected visual acuity; PVR, proliferative vitreoretinopathy.

Comparison was made for the eyes with single surgery anatomical success and for the eyes that needed vitrectomy owing to the redetachment associated with proliferative vitreoretinopathy.

at-test.

'Fisher's exact test.

${ }^{c} \chi^{2}$-test. 


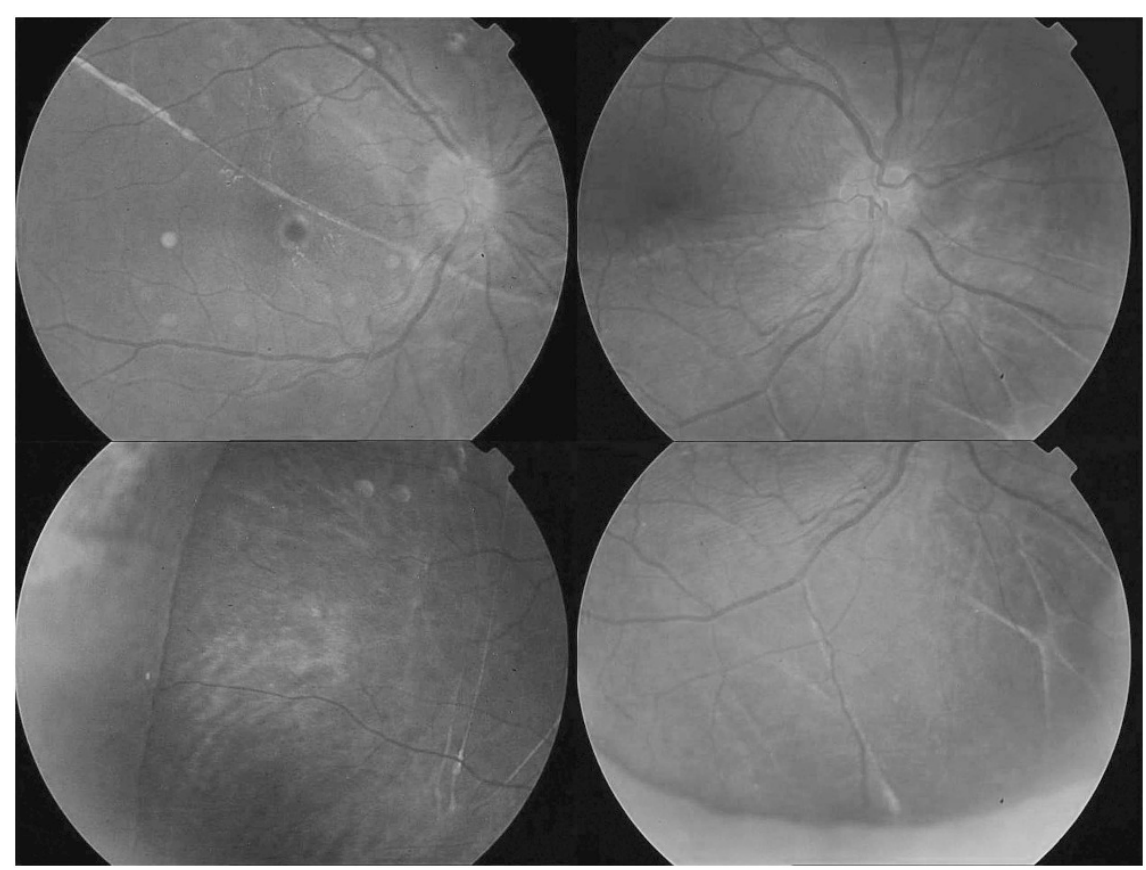

Figure 1 Fundus photography of eyes with retinal detachment associated with subretinal proliferation after successful scleral buckling surgery. The buckle effect is clearly visible in lower pictures.

in 37 eyes (84.1\%). Subretinal fluid drainage was performed in 42 eyes (95.5\%). Drainage was performed before tightening the buckling element. In 29 eyes (65.9\%), after creation of a $2-3 \mathrm{~mm}$ scleral incision and application of the choroidal diathermy, subretinal fluid was drained from a choroidal puncture using a 27 gauge needle. In other eyes (29.5\%), a 27 gauge needle was inserted directly into the subretinal space. Intraocular pressure (IOP) restored after tightening of the buckling element. In cases with significant increase in IOP after tightening of the buckling element, an anterior chamber tap was performed. No eye needed intravitreal gas injection. Intraoperative complications included retinal perforation with vitreous incarceration during subretinal fluid drainage in two eyes (4.5\%). In these cases, the perforation site was well placed over the buckle and no additional surgical modification was needed.

Immediately after the surgery, retina was reattached in all eyes. Figure 1 shows some examples of eyes successfully treated with scleral buckling alone. Five eyes $(11.3 \%)$ needed additional surgery for the treatment of retinal redetachment later in the course of the follow-up (Table 2). Therefore, single surgery success rate was $88.7 \%$. In four eyes (9.1\%), redetachment was associated with PVR and pars plana vitrectomy resulted in retinal reattachment. Scleral buckle revision surgery was successfully performed in the other eye. Retinal detachment anterior to the buckle was observed in one eye which remained unchanged until the last examination (24 months after surgery). A rise in IOP was found in three eyes (6.8\%); which was medically controlled in two eyes and needed cyclophotocoagulation in one eye. One eye needed pars plana vitrectomy for macular pucker. Postoperative barrier laser photocoagulation of the untreated or new breaks was performed in 11 eyes (25\%). At the end of follow-up, anatomical success defined as retinal reattachment posterior to the scleral buckle was found in all eyes (100\%).

No significant difference was found in preoperative and intraoperative data between eyes with single surgery anatomical success and those who needed pars plana vitrectomy for postoperative retinal detachment with PVR (Table 1).

BCVA was $1.5 \pm 0.9 \log$ MAR (range: $0.1-3 \log$ MAR) and $1.1 \pm 0.7 \log$ MAR (range: $0-3 \log$ MAR) before and after surgery, respectively $(P<0.001)$. An improvement in BCVA of $>2$ lines was found in 23 eyes (52.2\%) and worsening of BCVA of $>2$ lines was observed in 2 eyes (4.5\%). Complete records of pre and postoperative refractive error measurements were present for 20 eyes. Mean spherical equivalent refractive error was $-2.00 \pm 4.6$ and $-2.4 \pm 6.7$ diopters before and after surgery, respectively $(P=0.7)$.

\section{Discussion}

Subretinal proliferation has been reported in 3-15.5\% of eyes with uncomplicated RRD and $47 \%$ of eyes with RRD associated with PVR. ${ }^{9-11}$ Despite this prevalence, it has received relatively little attention in the literature. Miura and Ideta ${ }^{9}$ reported atrophic retinal breaks, young age, 


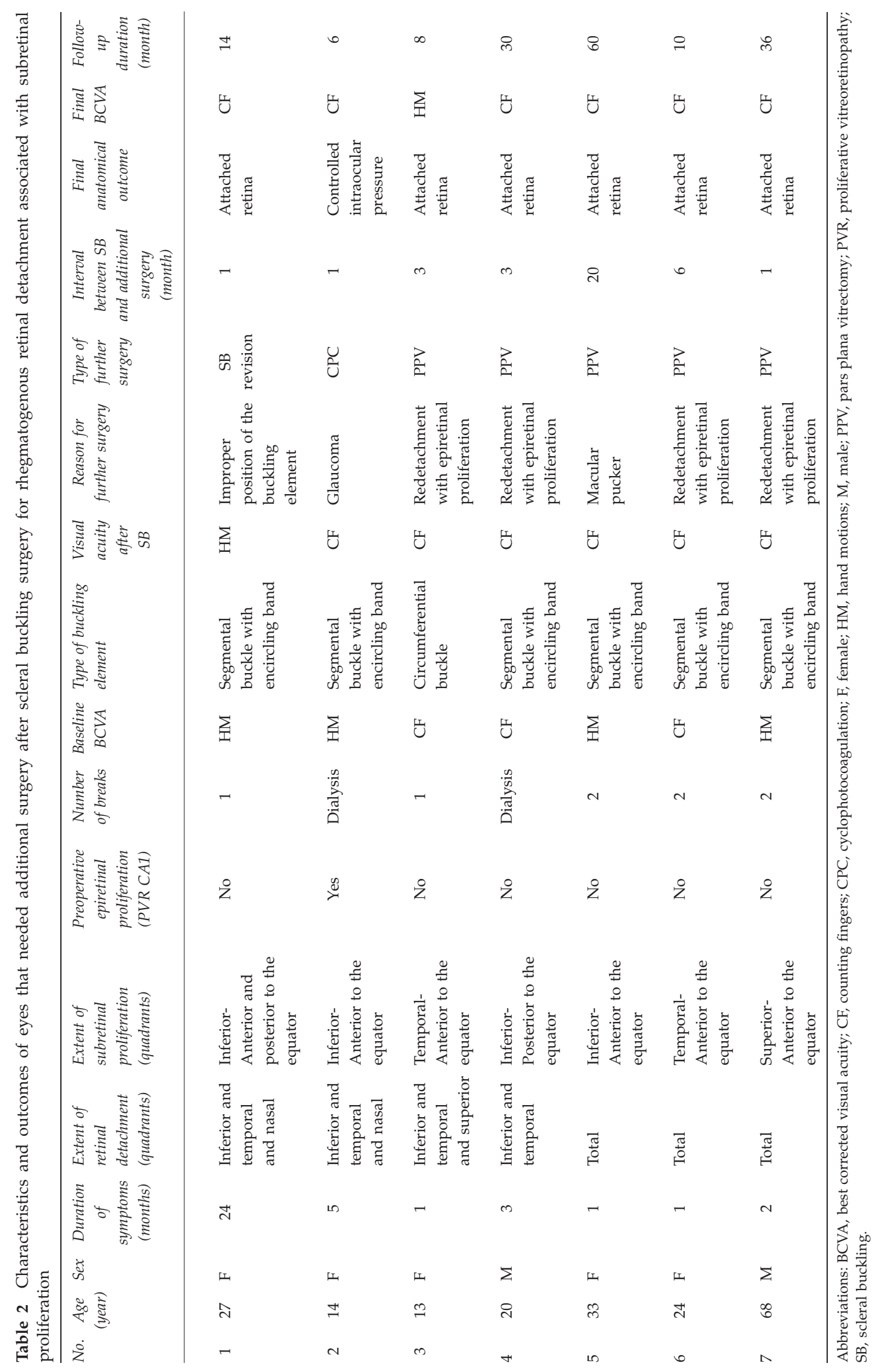


greater number of detached quadrants, and long standing RRD as the factors significantly associated with subretinal proliferations. Wallyn and Hilton ${ }^{11}$ reported an increase in the incidence of subretinal proliferation with the duration of RRD, from $0.8 \%$ in cases $<1$ month to $22 \%$ in cases with more than 2 years old.

Surgical management of eyes with RRD associated with PVR depends on the location and extent of membranes. Pars plana vitrectomy is needed for eyes with posterior and extensive anterior epiretinal proliferations with or without subretinal strands to remove the contractile membranes and release the resultant retinal shortening. Lewis et $a l^{10}$ reported that during vitrectomy for PVR with pre- and sub-retinal proliferations, only $28 \%$ of subretinal strands required special surgical therapy such as removal or transection, and the remainder did not interfere with conventional reattachment maneuvers. Moreover, the visual prognosis was reasonably good in the majority of patients that did not require removal or transection of the strands. On the other hand, if subretinal strand removal was necessary, the anatomic and visual outcomes were relatively poor with only $65 \%$ chance of final success and $20 \%$ chance of return to the ambulatory vision.

The treatment of choice in eyes with PVR associated with isolated subretinal proliferations (ie, without preretinal membranes) is not clear. Wallyn and Hilton ${ }^{11}$ reported retinal reattachment rate of $95 \%$ with scleral buckling surgery in 20 eyes with isolated subretinal proliferation. Yao et $a l^{12}$ reported results of scleral buckling surgery in 40 eyes with RRD and subretinal proliferation. Four eyes in their series had a small local preretinal membrane but without evidence of a starfold. In two eyes, the retina was not reattached and vitrectomy was performed. In one eye, the surgery was terminated after a narrow funnel-shaped RD was more clearly visualized after cataract surgery. The single surgery anatomical success was $90 \%$. Our results compare favorably with those reported by Yao et al, ${ }^{12}$ with a single surgery anatomical success rate of $88.7 \%$. In our study, however, the retina was attached in all eyes immediately after surgery. The reason for redetachment was the development of PVR in four eyes and a missed retinal break in another eye. Moreover, the scleral buckling surgery was successful in all eyes with PVR CA1 at the baseline. In these eyes, retinal reattachment and closure of retinal breaks resulted in the arrest of the epiretinal proliferation process, and the placement of anterior PVR on an encircling buckle, effectively released the retinal traction exerted by the epiretinal proliferation. There is no standard grading system for classification of the severity of the subretinal proliferation. We arbitrarily graded the subretinal proliferation based on the quadrants of proliferation. No correlation was found between the number of involved quadrants and the need for additional vitrectomy.

Pars plana vitrectomy in patients with RRD associated with subretinal proliferations requiring subretinal surgery may be associated with significant intraoperative complications including choroidal or retinal hemorrhage, subretinal air, and unplanned extension of the retinotomies. ${ }^{10}$ Moreover, long standing intraocular tamponade with silicone oil or C3F8 is needed at the conclusion of vitrectomy. These patients are typically young and intraocular tamponade may result in significant lens opacity necessitating the cataract surgery. On the other hand, scleral buckling surgery results in high anatomical success rate and remains a viable option for the treatment of RRD associated with subretinal proliferations with or without minimal anterior PVR. Previous studies have shown various changes in refractive error after scleral buckling surgery depending on the preoperative refractive state, surgical approach, buckle height, and so on. ${ }^{16}$ We found statistically similar amount of refractive error before and after surgery. Nevertheless, laser refractive surgery may be safely performed after stabilization of refractive error in the eyes with buckle-induced refractive changes. ${ }^{17}$

Our study has several limitations. The sample size was small. Statistically non-significant findings may be attributable to the sample size. Also, the study was retrospective and uncontrolled. In our center, scleral buckling is the preferred surgery for eyes with RRD associated with subretinal proliferation without significant preretinal proliferation. However, some eyes may undergo pars plana vitrectomy based on the surgeon's preference. We did not evaluate the outcomes of patients with subretinal proliferation who underwent pars plana vitrectomy surgery. Despite these limitations, this is the largest study specifically addressing the success of scleral buckling surgery in a homogenous group of patients with RRD associated with subretinal proliferations.

\section{Summary}

\section{What was known before}

- Subretinal proliferation is currently classified as high grade proliferative vitreoretinopathy. Pars plana vitrectomy and removal of contractile membranes is generally needed for a successful retinal reattachment surgery in patients with advanced proliferative retinopathy.

\section{What this study adds}

- Scleral buckling surgery is highly successful in eyes with rhegmatogenous retinal detachment associated with subretinal proliferation and no or minimal epiretinal proliferation. 


\section{Conflict of interest}

The authors declare no conflict of interest.

\section{References}

1 Heimann H, Bartz-Schmidt KU, Bornfeld N, Weiss C, Hilgers RD, Foerster $\mathrm{MH}$ et al. Scleral buckling versus primary vitrectomy in rhegmatogenous retinal detachment: a prospective randomized multicenter clinical study. Ophthalmology 2007; 114: 2142-2154.

2 Ahmadieh H, Moradian S, Faghihi H, Parvaresh MM, Ghanbari H, Mehryar M et al. Anatomic and visual outcomes of scleral buckling versus primary vitrectomy in pseudophakic and aphakic retinal detachment: six-month follow-up results of a single operation-report no. 1 . Ophthalmology 2005; 112: 1421-1429.

3 Sharma YR, Karunanithi S, Azad RV, Vohra R, Pal N, Singh DV et al. Functional and anatomic outcome of scleral buckling versus primary vitrectomy in pseudophakic retinal detachment. Acta Ophthalmol Scand 2005; 83: 293-297.

4 Sun Q, Sun T, Xu Y, Yang XL, Xu X, Wang BS et al. Primary vitrectomy versus scleral buckling for the treatment of rhegmatogenous retinal detachment: a meta-analysis of randomized controlled clinical trials. Curr Eye Res 2012; 37: 492-499.

5 Soni C, Hainsworth DP, Almony A. Surgical management of rhegmatogenous retinal detachment: a meta-analysis of randomized controlled trials. Ophthalmology 2013; 120: 1440-1447.

6 Feltgen N, Weiss C, Wolf S, Ottenberg D, Heimann H. SPR Study Group. Scleral buckling versus primary vitrectomy in rhegmatogenous retinal detachment study (SPR Study): recruitment list evaluation. Study report no. 2. Graefes Arch Clin Exp Ophthalmol 2007; 245: 803-809.
7 Pastor JC, de la Rúa ER, Martín F. Proliferative vitreoretinopathy: risk factors and pathobiology. Prog Retin Eye Res 2002; 21: 127-144.

8 Machemer R, Aaberg TM, Freeman HM, Irvine AR, Lean JS, Michels RM. An updated classification of retinal detachment with proliferative vitreoretinopathy. Am J Ophthalmol 1991; 112: 159-165.

9 Miura M, Ideta H. Factors related to subretinal proliferation in patients with primary rhegmatogenous retinal detachment. Retina 2000; 20(5): 465-468.

10 Lewis H, Aaberg TM, Abrams GW, McDonald HR, Williams GA, Mieler WF. Subretinal membranes in proliferative vitreoretinopathy. Ophthalmology 1989; 96: 1403-1414; discussion 1414-5.

11 Wallyn RH, Hilton GF. Subretinal fibrosis in retinal detachment. Arch Ophthalmol 1979; 97(11): 2128-2129.

12 Yao Y, Jiang L, Wang Z, Zhang M. Scleral Buckling Procedures for Longstanding or Chronic Rhegmatogenous Retinal Detachment with Subretinal Proliferation. Ophthalmology 2006; 113: 821-825.

13 Tabandeh H, Callejo SA, Rosa Jr RH, Flynn Jr HW. Subretinal "napkin-ring" membrane in proliferative vitreoretinopathy. Arch Ophthalmol 2000; 118(9): 1287-1289.

14 Holladay JT. Visual acuity measurements. J Cataract Refract Surg 2004; 30: 287-290.

15 Rodríguez de la Rúa E, Martínez V, Aragón J, Sanabria RM, Giraldo A, Mayo A et al. Clinical risk factors for postoperative proliferative vitreoretinopathy (PVR). A prospective study. Arch Soc Esp Oftalmol 2003; 78(2): 91-97.

16 Randleman JB, Hewitt SM, Stulting RD. Refractive changes after posterior segment surgery. Ophthalmol Clin North Am 2004; 17(4): 521-526.

17 Holopainen JM, Vuori E, Moilanen JA, Zalentein WN. Tervo TM.Excimer laser refractive correction of myopia after episcleral buckling for rhegmatogenous retinal detachment. J Cataract Refract Surg 2007; 33(10): 1744-1749. 